\title{
Prognosis of hemodialysis patients with progressive aortic stenosis: a prospective cohort study
}

\author{
Yoriko Horiguchi ${ }^{*} \mathbb{0}$, Kaoru Uemura ${ }^{2}$, Naoyoshi Aoyama ${ }^{3}$, Shinichi Nakajima ${ }^{4}$, Tomoki Asai ${ }^{4}$, \\ Sachiko Motohashi ${ }^{4}$, Manae Harada ${ }^{5},{\text { Maoko } l d a^{4} \text { and Atsushi Yoshida }}^{4}$
}

\begin{abstract}
Background: Whether progressive mild to moderate aortic stenosis in hemodialysis patients influences their prognosis has not been elucidated. This prospective cohort study explored whether progressive aortic stenosis predicted the rate of cardiac events and mortality in those patients.

Methods: A total of 283 consecutive hemodialysis patients (no aortic stenosis, 248; progressive aortic stenosis, 35) underwent echocardiography for assessment of aortic stenosis, with a median follow-up period of 4.1 years. Study endpoints were cardiac events, all-cause mortality, and cardiac death. Kaplan-Meier analysis and multivariate Cox proportional hazard analysis were performed to estimate cardiac events, all-cause mortality, and cardiac death.
\end{abstract}

Results: Cumulative cardiac event rate, all-cause mortality rate, and the rate of cardiac death at 3-year follow-up were $44.9 \%, 40.5 \%$, and $26.4 \%$ in patients with progressive aortic stenosis and $22.1 \%, 19.0 \%$, and $7.5 \%$ in those without aortic stenosis, respectively. Kaplan-Meier analysis demonstrated the cumulative rates of cardiac events and all-cause mortality. And cardiac death was significantly higher in patients with progressive aortic stenosis than in those without aortic stenosis. Multivariate Cox proportional hazard analysis revealed that progressive aortic stenosis was predictive of cardiac events (adjusted hazard ratio 2.47; 95\% confidence interval 1.38-4.39) and cardiac death (adjusted hazard ratio $4.21 ; 95 \%$ confidence interval $2.10-8.46$ ). Age, physical activity, C-reactive protein, and serum albumin levelsbut not progressive aortic stenosis—-predicted all-cause mortality.

Conclusions: The rates of cardiac events and cardiac death were higher in hemodialysis patients with progressive aortic stenosis than in those without aortic stenosis. Furthermore, progressive aortic stenosis predicted cardiac events and cardiac death. Compared with those without aortic stenosis, patients with progressive aortic stenosis had higher all-cause mortality, which was related to their comorbidities.

Trial registration This study was retrospectively registered with University Hospital Medical Information Network Clinical Trials Registry (registration number, UMIN 000024023) at September 12th, 2016.

Keywords: Aortic stenosis, Cardiac death, Cardiac events, Hemodialysis, Mortality

*Correspondence: dzq04361@nifty.com

1 Department of Cardiology, Sagami Circulatory Organ Clinic, 4-21-15 Sounan, Minami-ku, Sagamihara, Kanagawa 252-0312, Japan

Full list of author information is available at the end of the article

\section{Introduction}

Patients on hemodialysis often develop aortic stenosis (AS) after aortic valve calcification $[1,2]$. Severe AS is present in 4 to $13 \%$ of patients undergoing hemodialysis and is correlated with their poor survival $[1,3,4]$. In the general population, patients with progressive mild to original author(s) and the source, provide a link to the Creative Commons licence, and indicate if changes were made. The images or other third party material in this article are included in the article's Creative Commons licence, unless indicated otherwise in a credit line to the material. If material is not included in the article's Creative Commons licence and your intended use is not permitted by statutory regulation or exceeds the permitted use, you will need to obtain permission directly from the copyright holder. To view a copy of this licence, visit http://creativecommons.org/licenses/by/4.0/. The Creative Commons Public Domain Dedication waiver (http://creativeco mmons.org/publicdomain/zero/1.0/) applies to the data made available in this article, unless otherwise stated in a credit line to the data. 
moderate AS have a higher rate of cardiovascular events than those without AS, and moderate AS is associated with increased all-cause mortality [5-8]. Because of its association with cardiac valvular calcification, which influences the prognosis in hemodialysis patients, progressive AS may also influence the prognosis in hemodialysis patients. Knowing the prognosis of patients with progressive AS is crucial to managing them appropriately $[3,9]$. Whether progressive AS in hemodialysis patients influences their rate of cardiac events and mortality has not been elucidated. The aim of this prospective cohort study was to explore the prognostic influence of progressive AS on morbidity related to cardiac events and mortality in hemodialysis patients.

\section{Materials and methods}

\section{Study population and echocardiography}

This single-center, prospective cohort study was conducted in accordance with the ethical principles for medical research involving human subjects as delineated in the Declaration of Helsinki. This study was approved by the institutional review board of our institution, and written informed consent was obtained from each participant.

A total of 375 consecutive patients with end-stage renal disease who underwent maintenance hemodialysis at our institution from February 2016 through November 2017 were enrolled in our study. We excluded 78 patients who did not give informed consent and 8 patients who had undergone aortic valve replacement. Therefore, 289 patients underwent transthoracic echocardiography in this study.

We performed echocardiography of the patients by using an Aplio a Verifia system (Canon Medical Systems Corporation, Tochigi, Japan) according to the guidelines released by the American Society of Echocardiography and the European Association of Cardiovascular Imaging $[10,11]$. Left ventricular volume and ejection fraction were calculated by using the biplane disk summation method [10]. We measured the aortic valve area (AVA) in each patient with AS, which was calculated by using the continuity equation, peak aortic jet velocity (aortic $V_{\max }$ ), mean pressure gradient across the aortic valve (mean $\Delta P$ ), and stroke volume index (SVI) [11]. We classified the severity of AS according to the 2014 American Heart Association/American College of Cardiology guidelines for the management of patients with valvular heart disease as follows: no AS, aortic $V_{\max }<2 \mathrm{~m} / \mathrm{s}$; progressive AS, AVA $>1.0 \mathrm{~cm}^{2}$ or AVA $\leq 1.0 \mathrm{~cm}^{2}$ with $\mathrm{SVI} \geq 35 \mathrm{~mL} /$ $\mathrm{m}^{2}$, aortic $V_{\max }$ of $2.0-3.9 \mathrm{~m} / \mathrm{s}$, or mean $\Delta P<40 \mathrm{mmHg}$; severe AS (high gradient), AVA $\leq 1.0 \quad \mathrm{~cm}^{2}$, aortic $V_{\max } \geq 4.0 \mathrm{~m} / \mathrm{s}$ or mean $\Delta P \geq 40 \mathrm{mmHg}$; and severe AS (low gradient), AVA $\leq 1.0 \mathrm{~cm}^{2}$ with $\mathrm{SVI}<35 \mathrm{~mL} / \mathrm{m}^{2}$, aortic $V_{\max }<4.0 \mathrm{~m} / \mathrm{s}$, and mean $\Delta P<40 \mathrm{mmHg}$ [12]. Six patients were diagnosed to have severe AS and were therefore excluded from the study. Consequently, 283 patients (no AS, 248; progressive AS, 35) were evaluated in this study.

\section{Assessment of clinical status}

We assessed participants' physical activity by using the Specific Activity Scale (SAS) questionnaire within a week before or after the day on which echocardiography was performed [13]. The SAS score (metabolic equivalents [METs]) was determined as the lowest intensity of activity that the patients could not perform. We then asked the patients whether they had had exercise-related symptoms of dyspnea, chest pain, or syncope within the past 2 months.

Blood tests were performed within 2 weeks before or after the day when echocardiography was done. Blood samples were collected before the first hemodialysis procedure of the week. We examined the medical records of patients for history of admission on account of ischemic heart disease or heart failure.

Peripheral artery disease (PAD) was diagnosed in patients with abnormal ankle brachial index (i.e., $\leq 0.9$ ) or toe brachial index (i.e., $\leq 0.6$ ), in those with lower limb arterial echography showing $>50 \%$ stenosis, and those with a history of peripheral vascular revascularization or limb amputation due to PAD [14].

\section{Endpoints}

Patient follow-up commenced on the day when echocardiography was performed and continued until January 2021. We included patients who were lost to follow-up in the analyses. We noted the timing of cardiac events and all-cause mortality in the study patients. Cardiac events included aortic valve replacement, hospital admission due to heart failure, acute coronary syndrome, coronary artery revascularization, resuscitated arrhythmia and cardiac arrest, and cardiac death during the follow-up period. Cardiac death was defined as death due to heart failure, acute myocardial infarction, or fatal arrhythmia or sudden death.

\section{Statistical analysis}

The statistical power of the survival analysis at the twosided significance level of 0.05 was 0.91 when the 35 patients with progressive AS had a $22 \%$ higher 3-year event rate than the 248 patients without AS.

Continuous values were expressed as means \pm 1 standard deviation. To assess patient characteristics, we used the unpaired or paired $t$-test, one-way analysis of variance, Tukey test, and nonparametric tests (MannWhitney $U$ test or Kruskal-Wallis test) for continuous 
variables and the Chi-square test or Fisher exact test for categorical variables. The rates of all-cause mortality, cardiac events, and cardiac death were analyzed by using the Kaplan-Meier method with log-rank testing. We used Cox proportional hazard analysis to assess which variables were related to all-cause mortality, cardiac events, and cardiac death. First, we conducted univariate Cox proportional hazard analysis. We analyzed the independent variables of age, male gender, body mass index, length of time on dialysis, SAS score, presence of progressive AS, PAD, history of diabetes, hypertension, dyslipidemia, ischemic heart disease, heart failure, cerebrovascular disease, present tobacco use, hemoglobin, serum level of albumin, adjusted calcium, phosphorus, calcium-phosphorus product, C-reactive protein, and low-density lipoprotein. Then, we performed multivariate Cox proportional hazard analysis adjusted for statistically significant independent variables (i.e., $P<0.05)$ in the univariate Cox proportional hazard analysis. To evaluate prognostic factors affecting the onset of cardiac death, we performed stepwise multivariate Cox proportional hazard analysis.

A $P$ value of less than 0.05 was considered to indicate statistical significance. All statistical analyses were performed by using IBM SPSS Statistics (version 22, IBM Corporation, Armonk, NY, USA) and EZR version 1.41 ( $\mathrm{R}$ version 3.6.1) [15].

\section{Results}

\section{Patients}

Study participants $(n=283)$ were followed for a median period of 4.1 years (interquartile range 2.8-4.4 years). Fifteen (5.3\%) patients were lost to follow-up during the study period. A total of 190 (96.0\%) and 144 (72.7\%) of the 198 surviving patients had a minimum follow-up period of 3 and 4 years, respectively.

The baseline characteristics of the study patients are shown in Table 1. No patient had bicuspid aortic valve. Patients with progressive AS were significantly older and had lower SAS scores and higher prevalence of PAD than those without AS (Table 1). The frequency of the exercise-induced symptoms did not differ significantly between these patient groups (Table 1).

Echocardiographic data showed that the left ventricular volume and ejection fractions of patients with progressive AS were similar to those without AS (Table 2). The ratio between early mitral inflow velocity and mitral annular early diastolic velocity $\left(E / e^{\prime}\right)$ was measured in 165 patients (patients without AS, 146; progressive AS, 19). E/e $e^{\prime}$ was higher in those with progressive AS $(17.0 \pm 8.4)$ than those without AS $(11.9 \pm 4.6$, $P=0.015)$.

\section{Follow-up and endpoints}

During follow-up, 5 (1.8\%) patients developed severe AS and $2(0.7 \%)$ underwent aortic valve replacement. In addition, $1(0.4 \%)$ patient developed coronavirus disease 2019 and recovered without cardiovascular complication. A total of $85(30.0 \%)$ patients (with progressive AS, 18; without AS, 67) experienced cardiac events. These cardiac events included hospitalization for heart failure for $44(15.5 \%)$ patients, coronary revascularization and acute coronary syndrome in 29 (10.2\%), sudden death in $6(2.1 \%)$, fatal or resuscitated arrhythmia in $4(1.4 \%)$, and aortic valve intervention in $2(0.7 \%)$. A total of $86(30.4 \%)$ patients (with progressive AS, 19; without AS, 67) died due to various causes during follow-up (cardiac death, 38 [13.4\%]; infection, 25 [8.8\%]; malignancy, 7 [2.5\%]; gastrointestinal bleeding, 2 [0.7\%]; lethal hyperkalemia, 1 [0.4\%]; stroke, 1 [0.4\%]; extrinsic death, 2 [0.7\%]; cause unknown, 10 [3.5\%]). Cardiac death occurred in 12 patients with progressive AS and 26 in those without AS, respectively. The causes of the cardiac death were: heart failure in 17 (6.0\%) patients, sudden death in 10 (3.5\%), acute myocardial infarction in $8(2.8 \%)$, and fatal arrhythmia in 3 (1.1\%).

The cumulative cardiac event rate at the 3-year follow-up was $44.9 \%$ in patients with progressive AS and $22.1 \%$ in those without AS (Fig. 1). Kaplan-Meier analysis revealed that the cumulative cardiac event rate was higher in patients with progressive AS than in those without AS (the 3-year follow-up, $P=0.002$; the entire follow-up, $P<0.001$ ) (Fig. 1). The patients with progressive AS had a higher cumulative rate of composite coronary events than those without AS $(28.2 \%$ vs. $9.1 \%$ at the 3 -year follow-up, $P=0.003 ; 69.0 \%$ vs. $23.3 \%$ at the end of follow-up, $P=0.004$ ), but their cumulative rate of heart failure hospitalization was comparable to that of those without AS $(22.4 \%$ vs. $13.3 \%$ at the 3 -year follow-up, $P=0.105 ; 33.5 \%$ vs. $27.4 \%$ at the final follow-up, $P=0.120)$.

The cumulative incidence of all-cause mortality at the 3-year follow-up was $40.5 \%$ in patients with progressive AS and $19.0 \%$ in those without AS. Similarly, KaplanMeier analysis demonstrated that the cumulative rate of all-cause mortality was higher in the patients with progressive AS than in those without AS (the 3-year followup, $P<0.001$; the entire follow-up, $P<0.001$ ) (Fig. 2). The cumulative rate of cardiac death at the 3-year followup was $26.4 \%$ in patients with progressive AS and $7.5 \%$ in those without AS. Kaplan-Meier analysis revealed a higher cumulative rate of cardiac death in patients with progressive AS than in those without AS (the 3-year follow-up, $P<0.001$; the entire follow-up, $P<0.001$ ) (Fig. 3). Throughout follow-up among the patients with progressive AS, cardiac death occurred in 6 of the 9 patients with 
Table 1 Baseline characteristics of the study patients

\begin{tabular}{|c|c|c|c|}
\hline Parameter & Patients with progressive AS & Patients without AS & $P$ \\
\hline Number of patients & 35 & 248 & \\
\hline Age (year) & $74 \pm 11$ & $67 \pm 12$ & $<0.001$ \\
\hline Male gender, $n(\%)$ & $20(57)$ & $144(58)$ & 1.00 \\
\hline Body mass index $\left(\mathrm{kg} / \mathrm{m}^{2}\right)$ & $21.0 \pm 4.0$ & $21.9 \pm 4.6$ & 0.27 \\
\hline Length of time on dialysis (year) & $12 \pm 9$ & $9 \pm 9$ & 0.08 \\
\hline Specific Activity Scale (METs) & $3.5 \pm 2.0$ & $4.3 \pm 2.1$ & 0.03 \\
\hline \multicolumn{4}{|l|}{ Exercise-related symptoms, $n$ (\%) } \\
\hline Syncope & $5(14)$ & $16(7)$ & 0.16 \\
\hline Chest pain & $7(20)$ & $54(22)$ & 1.00 \\
\hline Shortness of breath & $12(34)$ & $65(26)$ & 0.32 \\
\hline Symptom, any of the above & $12(34)$ & $98(40)$ & 0.59 \\
\hline \multicolumn{4}{|l|}{ Coexisting conditions, $n$ (\%) } \\
\hline Diabetes & $13(37)$ & $107(43)$ & 0.59 \\
\hline Hypertension & $30(86)$ & $192(77)$ & 0.38 \\
\hline Dyslipidemia & $14(40)$ & $101(41)$ & 1.00 \\
\hline Ischemic heart disease & $9(26)$ & $60(24)$ & 0.84 \\
\hline Heart failure & $11(31)$ & $45(18)$ & 0.07 \\
\hline Atrial fibrillation & $3(8)$ & $26(11)$ & 1.00 \\
\hline Peripheral artery disease & $20(57)$ & $75(30)$ & 0.004 \\
\hline History of PTA or limb amputation & $8(23)$ & $34(14)$ & 0.20 \\
\hline Cerebrovascular disease & $9(26)$ & $51(21)$ & 0.51 \\
\hline Past or present tobacco use & $15(43)$ & $102(41)$ & 0.86 \\
\hline \multicolumn{4}{|l|}{ Medications, $n(\%)$} \\
\hline ACE inhibitor & $1(3)$ & $9(4)$ & 1.00 \\
\hline ARB & $14(40)$ & $93(38)$ & 0.85 \\
\hline Calcium channel blocker & $22(63)$ & $132(53)$ & 0.37 \\
\hline Beta blocker & $10(29)$ & $91(37)$ & 0.45 \\
\hline Statin & $14(40)$ & $101(41)$ & 1.00 \\
\hline Vitamin $D_{3}$ & $14(40)$ & $127(51)$ & 0.28 \\
\hline Phosphate binder & $13(37)$ & $88(35)$ & 0.85 \\
\hline Calcimimetics & $5(14)$ & $29(12)$ & 0.59 \\
\hline Calcium carbonate & $26(74)$ & $160(65)$ & 0.34 \\
\hline \multicolumn{4}{|l|}{ Blood test results } \\
\hline Hemoglobin (g/dL) & $10.7 \pm 1.3$ & $10.8 \pm 1.1$ & 0.72 \\
\hline Albumin (g/dL) & $3.5 \pm 0.4$ & $3.6 \pm 0.4$ & 0.08 \\
\hline Adjusted calcium (mg/dL) & $9.2 \pm 0.6$ & $9.0 \pm 0.8$ & 0.12 \\
\hline Phosphorus (mg/dL) & $5.1 \pm 1.4$ & $5.4 \pm 1.3$ & 0.27 \\
\hline Calcium-phosphorus product $\left(\mathrm{mg}^{2} / \mathrm{dL}^{2}\right)$ & $47.9 \pm 12.7$ & $47.2 \pm 13.2$ & 0.78 \\
\hline C-reactive protein (mg/dL) & $2.1 \pm 5.4$ & $0.6 \pm 1.9$ & 0.18 \\
\hline LDL cholesterol (mg/dL) & $81 \pm 24$ & $81 \pm 22$ & 0.68 \\
\hline
\end{tabular}

ACE angiotensin-converting enzyme, ARB angiotensin II receptor blocker, $A S$ aortic stenosis, $L D L$ low-density lipoprotein, METs metabolic equivalents, PTA percutaneous transluminal angioplasty

a history of ischemic heart disease, in 4 of the 11 patients with a history of heart failure, and in 3 of the 8 patients with no history of ischemic heart disease or heart failure.

Univariate Cox proportional hazard analysis identified the following independent variables as predictors of cardiac events: age, male gender, body mass index, SAS score, progressive AS, history of diabetes, ischemic heart disease, heart failure, and PAD. Multivariate Cox proportional hazard analysis revealed that independent predictors of cardiac events were history of ischemic heart disease (adjusted hazard ratio 2.50; 95\% confidence interval $[\mathrm{CI}] 1.55-4.03 ; P<0.001)$ and presence of progressive 
Table 2 Echocardiographic data of the study patients

\begin{tabular}{|c|c|c|c|}
\hline Parameter & $\begin{array}{l}\text { Patients with } \\
\text { progressive } \\
\text { AS }\end{array}$ & Patients without AS & $P$ \\
\hline Number of patients & 35 & 248 & \\
\hline LVEDVI $\left(\mathrm{mL} / \mathrm{m}^{2}\right)$ & $105 \pm 22$ & $97 \pm 31$ & 0.12 \\
\hline LVESVI $\left(\mathrm{mL} / \mathrm{m}^{2}\right)$ & $50 \pm 20$ & $45 \pm 20$ & 0.21 \\
\hline LVEF (\%) & $56 \pm 13$ & $56 \pm 14$ & 0.93 \\
\hline LADs (mm) & $40 \pm 7$ & $39 \pm 20$ & 0.62 \\
\hline IVSd (mm) & $10 \pm 2$ & $10 \pm 3$ & 0.96 \\
\hline PWd (mm) & $10 \pm 2$ & $10 \pm 2$ & 0.98 \\
\hline$E / A$ & $0.85 \pm 0.30$ & $0.86 \pm 0.93$ & 0.89 \\
\hline $\operatorname{AVA}\left(\mathrm{cm}^{2}\right)$ & $1.5 \pm 0.4$ & NA & \\
\hline Mean aortic $\mathrm{PG}(\mathrm{mmHg})$ & $18.7 \pm 17.8$ & NA & \\
\hline Aortic $V_{\max }(\mathrm{cm} / \mathrm{s})$ & $269.4 \pm 43.1$ & NA & \\
\hline
\end{tabular}

$A S$ aortic stenosis, $A V A$ aortic valve area, $E / A$ the ratio of the early and late diastolic trans mitral flow velocity, IVS $d$ interventricular septum thickness at end diastole, LADs left atrial dimension at end systole, LVEDVI left ventricular end-diastolic volume index, LVEF left ventricular ejection fraction, LVESVI left ventricular end-systolic volume index, NA not available, $P G$ pressure gradient, $P W d$ posterior left ventricular wall thickness at end diastole, $V_{\max }$ maximum velocity

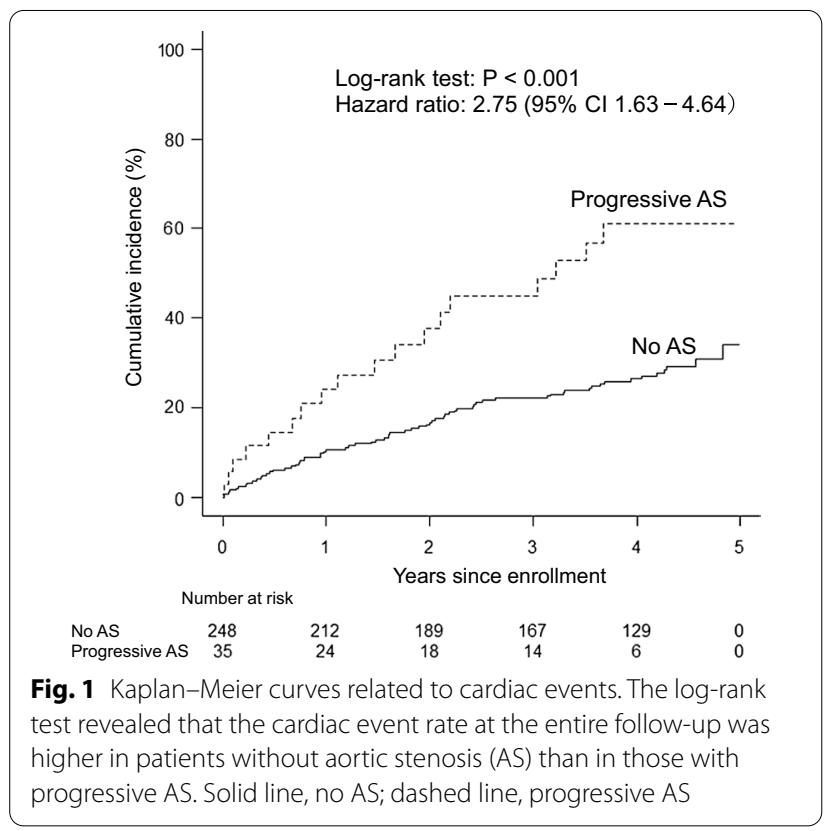

AS (adjusted hazard ratio 2.47; 95\% CI 1.38-4.39; $P=0.002$ ) (Table 3 ).

Univariate Cox proportional hazard analysis demonstrated that independent predictors of all-cause mortality were age, SAS score, progressive AS, history of ischemic heart disease, PAD, serum albumin level, and C-reactive protein level. Multivariate Cox proportional hazard analysis showed that age, SAS score, and serum albumin and
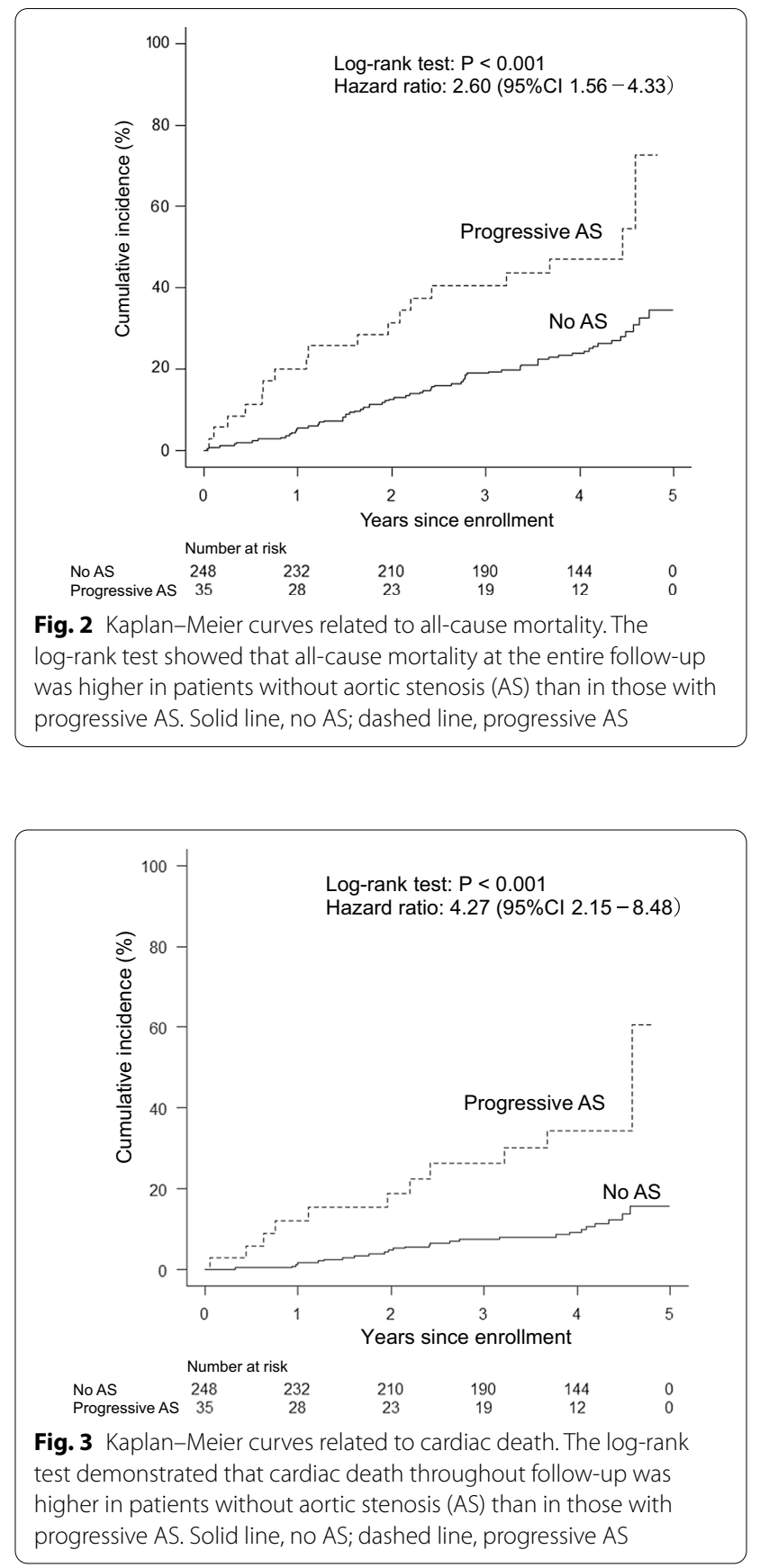

C-reactive protein levels were independent predictors for all-cause mortality (Table 4).

Univariate Cox proportional hazard analysis regarding prediction of cardiac death revealed that SAS score, presence of progressive AS, history of diabetes, history of ischemic heart disease, and serum levels of albumin and C-reactive protein were significant independent variables. Stepwise multivariate Cox proportional hazard analysis found that SAS score, progressive AS, and 
Table 3 Multivariate Cox proportional hazard analysis regarding prediction of cardiac events

\begin{tabular}{lllc}
\hline Variable & $\begin{array}{l}\text { Adjusted } \\
\text { hazard } \\
\text { ratio }\end{array}$ & $\mathbf{9 5 \%} \mathrm{Cl}$ & $\boldsymbol{P}$ \\
& 1.02 & $1.00-1.04$ & 0.13 \\
\hline Age (per 1 year) & 1.38 & $0.84-2.26$ & 0.20 \\
Male gender & 1.05 & $0.99-1.10$ & 0.09 \\
Body mass index (per 1 kg/m $\left.{ }^{2}\right)$ & 0.90 & $0.80-1.03$ & 0.12 \\
Specific Activity Scale score (per 1 & & & \\
MET) & 2.47 & $1.38-4.39$ & 0.002 \\
Progressive AS & 1.40 & $0.86-2.31$ & 0.18 \\
Diabetes & 2.50 & $1.55-4.03$ & $<0.001$ \\
Ischemic heart disease & 1.54 & $0.94-2.51$ & 0.08 \\
Heart failure & 0.89 & $0.53-1.49$ & 0.66 \\
Peripheral artery disease & & &
\end{tabular}

Other abbreviations as in Table 1

$\mathrm{Cl}$ confidence interval

Table 4 Multivariate Cox proportional hazard analysis regarding prediction of all-cause mortality

\begin{tabular}{lllc}
\hline Variable & $\begin{array}{l}\text { Adjusted } \\
\text { hazard } \\
\text { ratio }\end{array}$ & 95\% Cl & P \\
& 1.03 & $1.01-1.05$ & 0.009 \\
\hline Age (per 1 year) & 0.77 & $0.66-0.89$ & $<0.001$ \\
$\begin{array}{l}\text { Specific Activity Scale score (per 1 } \\
\text { MET) }\end{array}$ & 1.57 & $0.89-2.77$ & 0.12 \\
Progressive AS & 1.54 & $0.95-2.48$ & 0.077 \\
Ischemic heart disease & 1.10 & $0.69-1.77$ & 0.68 \\
Peripheral artery disease & 0.54 & $0.31-0.95$ & 0.032 \\
Serum albumin level (per 1 g/dL) & 1.10 & $1.03-1.19$ & 0.007 \\
C-reactive protein (per 1 mg/dL) & & & \\
\hline
\end{tabular}

Abbreviations as in Tables 1 and 3

Table 5 Stepwise multivariate Cox proportional hazard analysis regarding prediction of cardiac death

\begin{tabular}{|c|c|c|c|}
\hline Variable & $\begin{array}{l}\text { Adjusted } \\
\text { hazard ratio }\end{array}$ & $95 \% \mathrm{Cl}$ & $P$ \\
\hline $\begin{array}{l}\text { Specific Activity Scale score } \\
\text { (per } 1 \mathrm{MET} \text { ) }\end{array}$ & 0.70 & $0.58-0.85$ & $<0.001$ \\
\hline Progressive AS & 4.21 & $2.10-8.46$ & $<0.001$ \\
\hline Ischemic heart disease & 3.04 & $1.59-5.83$ & $<0.001$ \\
\hline
\end{tabular}

Abbreviations as in Tables 1 and 3

history of ischemic heart disease were independent predictors for cardiac death (Table 5).

\section{Discussion}

In this prospective cohort study, we compared the prognosis of maintenance hemodialysis patients with progressive AS with the prognosis of those without AS with a median follow-up period of 4.1 years. The present study has two main findings. First, the rates of cardiac events, all-cause mortality, and cardiac death were higher in patients with progressive AS than in those without AS. Second, the presence of progressive AS and history of ischemic heart disease independently predicted cardiac events and cardiac death. In contrast, progressive AS was not predictive of all-cause mortality.

Our study indicated that the cardiac event rate was higher in hemodialysis patients with progressive AS than in those without AS. Especially, the cumulative rate of cardiac death was more than three times higher in patients with progressive AS than in those without AS. Furthermore, progressive AS - together with a history of ischemic heart disease-was predictive of cardiac events and cardiac death in those patients. Although the mean left ventricular ejection fraction was comparable between patients with or without progressive AS, $E / e^{\prime}$ was significantly higher in those with progressive AS. Hence, diastolic dysfunction resulting from myocardial extracellular expansion and replacement fibrosis due to AS may cause an imbalance in myocardial oxygen supply and demand and thus influence the onset of cardiac events, including cardiac death [16]. Careful cardiovascular management and body fluid control are needed for patients with progressive AS to prevent cardiac events.

Our analysis revealed that the 3-year rates of cardiac events and cardiac mortality in hemodialysis patients with progressive AS were $44.9 \%$ and $26.4 \%$, respectively. Some studies have reported the risk of cardiac events in patients with progressive AS in the general population. For example, the cumulative incidence of heart failure hospitalization and aortic valve replacement at 3 years of follow-up were $47 \%$ in patients with moderate AS and reduced left ventricular ejection fraction [6]. In addition, the composite of ischemic cardiovascular events and aortic valve-related events occurred in $39 \%$ of asymptomatic patients with progressive AS during a median follow-up of 4.3 years [3]. In a large cohort study, the 5 -year rate of cardiovascular mortality was $31.6 \%$ in the patients with progressive AS [7]. Given these previous reports, the rates of cardiac events and cardiac mortality in hemodialysis patients with progressive AS may be comparable to those in the general population with progressive AS.

In our study, the 3-year all-cause mortality in hemodialysis patients with progressive AS was $40.5 \%$. This mortality may be higher than that of subjects with progressive AS in the general population (18-28\%) [6-8]. The present study found that the increased incidence of all-cause mortality in patients with progressive AS was not associated with a diseased aortic valve but instead was related to their comorbidities. Previous reports that assessed the general population 
obtained conflicting results regarding the involvement of progressive AS in all-cause death. Aortic $V_{\max }$ was an independent predictor of the composite of death and cardiac events in patients with moderate AS and a reduced left ventricular ejection fraction [6]. In a large cohort study, multivariate Cox proportional hazard analysis demonstrated that mean $\Delta P \geq 20 \mathrm{mmHg}$ was an independent predictor for all-cause mortality; however, this analysis was adjusted only for age and gender and disregarded other clinical risk factors [7]. Another group found that age and the presence of comorbidities predicted all-cause mortality in patients with moderate AS, but moderate AS itself did not predict it [8]. The results of that study were similar to ours, in which age, low physical activity, high C-reactive protein level, and hypoalbuminemia-all of which are known predictors for mortality in hemodialysis patients-predicted allcause mortality in our patients [8, 17-19]. We consider that hemodialysis patients with progressive AS should be aggressively managed for these risk factors because they could adversely affect the outcomes of future aortic valve interventions.

In our present study, the prevalence of exerciserelated symptoms was comparable between patients with and without progressive AS. Patients receiving hemodialysis often have these symptoms, probably because of excess body fluid, heart failure, or ischemic heart disease [3]. Therefore, discerning whether AS is a direct cause of these symptoms may be difficult.

The main limitation of this research was that it was a single-center study. In addition, the time interval between echocardiography and subsequent hemodialysis differed for each patient. The timing of hemodialysis might have variably influenced the cardiac hemodynamic condition.

\section{Conclusions}

In conclusion, compared with those without AS, hemodialysis patients with progressive AS had higher rates of cardiac events, cardiac death, and mortality. In addition, progressive AS was predictive of cardiac events and cardiac death in hemodialysis patients. The higher all-cause mortality in those with progressive AS was related to their comorbidities.

\section{Abbreviations}

Aortic $V_{\text {max }}$ : Peak aortic jet velocity; AS: Aortic stenosis; AVA: Aortic valve area; $E / e^{\prime}$ : The ratio between early mitral inflow velocity and mitral annular early diastolic velocity; Mean $\triangle P$ : Mean pressure gradient across the aortic valve; METs: Metabolic equivalents; PAD: Peripheral artery disease; SAS: Specific Activity Scale; SVI: Stroke volume index.
}

\section{Acknowledgements}

We are thankful to ELSS, Co., Ltd. for editing the draft of this manuscript.

\section{Authors' contributions}

YH designed this study, acquired, and analyzed the data. YH wrote the manuscript. KU contributed to the conception of this study and data acquisition. NA contributed to the conception of this study and revised the manuscript critically. SN, TA, SM, MH, and MI contributed to data acquisition. AY approved the study design. All authors read and approved the final manuscript.

\section{Funding}

None.

\section{Availability of data and materials}

The datasets generated and/or analyzed during this study are available from the corresponding author on reasonable request.

\section{Declarations}

\section{Ethics approval and consent to participate}

This study was conducted in accordance with the ethical principles for medical research involving human subjects as delineated in the Declaration of Helsinki and was approved by the institutional review board of Sagami Circulatory Organ Clinic (approval number: 2016-3). Written informed consent was obtained from each participant.

\section{Consent for publication}

Consent for publication was acquired from each participant.

\section{Competing interests}

The authors declare that they have no competing interests.

\section{Author details}

${ }^{1}$ Department of Cardiology, Sagami Circulatory Organ Clinic, 4-21-15 Sounan, Minami-ku, Sagamihara, Kanagawa 252-0312, Japan. ${ }^{2}$ Department of Clinical Laboratory, Sagami Circulatory Organ Clinic, Sagamihara, Kanagawa, Japan. ${ }^{3}$ Department of General Medicine, Kitasato University School of Medicine, Sagamihara, Kanagawa, Japan. ${ }^{4}$ Department of Internal Medicine, Sagami Circulatory Organ Clinic, Sagamihara, Kanagawa, Japan. ${ }^{5}$ Department of Rehabilitation, Sagami Circulatory Organ Clinic, Sagamihara, Kanagawa, Japan.

Received: 17 June 2021 Accepted: 29 August 2021

Published online: 08 September 2021

\section{References}

1. Matsuo H, Dohi K, Machida H, Takeuchi H, Aoki T, Nishimura H, et al. Echocardiographic assessment of cardiac structural and functional abnormalities in patients with end-stage renal disease receiving chronic hemodialysis. Circ J. 2018;82:586-95.

2. Ahmad Y, Bellamy MF, Baker CSR. Aortic stenosis in dialysis patients. Semin Dial. 2017;30:224-31.

3. Rattazzi M, Bertacco E, Del Vecchio A, Puato M, Faggin E, Pauletto P. Aortic valve calcification in chronic kidney disease. Nephrol Dial Transplant. 2013;28:2968-76.

4. Kawase Y, Taniguchi T, Morimoto T, Kadota K, Iwasaki K, Kuwayama A, et al. Severe aortic stenosis in dialysis patients. J Am Heart Assoc. 2017. https:// doi.org/10.1161/JAHA.116.004961.

5. Gerdts E, Rossebø AB, Pedersen TR, Cioff G, Lønnebakken MT, Cramariuc $D$, et al. Relation of left ventricular mass to prognosis in initially asymptomatic mild to moderate aortic valve stenosis. Circ Cardiovasc Imaging. 2015. https://doi.org/10.1161/CIRCIMAGING.115.003644.

6. van Gils L, Clavel MA, Vollema EM, Hahn RT, Spitzer E, Delgado V, et al. Prognostic implications of moderate aortic stenosis in patients with left ventricular systolic dysfunction. J Am Coll Cardiol. 2017;69:2383-92.

7. Strange G, Stewart S, Celermajer D, Prior D, Scalia GM, Marwick T, et al. Poor long-term survival in patients with moderate aortic stenosis. J Am Coll Cardiol. 2019;74:1851-63.

8. Delesalle G, Bohbot Y, Rusinaru D, Delpierre Q, Maréchaux S, Tribouilloy C. Characteristics and prognosis of patients with moderate aortic stenosis and preserved left ventricular ejection fraction. J Am Heart Assoc. 2019. https://doi.org/10.1161/JAHA.118.011036. 
9. Wang Z, Jiang A, Wei F, Chen H. Cardiac valve calcification and risk of cardiovascular or all-cause mortality in dialysis patients: a meta-analysis. BMC Cardiovasc Disord. 2018. https://doi.org/10.1186/s12872-018-0747-y.

10. Lang RM, Badano LP, Mor-Avi V, Afilalo J, Armstrong A, Ernande L, et al. Recommendations for cardiac chamber quantification by echocardiography in adults: an update from the American Society of Echocardiography and the European Association of Cardiovascular Imaging. Eur Heart J Cardiovasc Imaging. 2015;16:233-70.

11. Baumgartner H, Hung J, Bermejo J, Chambers JB, Edvardsen T, Goldstein $\mathrm{S}$, et al. Recommendations on the echocardiographic assessment of aortic valve stenosis: a focused update from the European Association of Cardiovascular Imaging and the American Society of Echocardiography. J Am Soc Echocardiogr. 2017;30:372-92.

12. Nishimura RA, Otto CM, Bonow RO, Carabello BA, Erwin JP 3rd, Guyton RA, ACC/AHA Task Force Members, et al. AHA/ACC Guideline for the management of patients with valvular heart disease: executive summary: a report of the American College of Cardiology/American Heart Association task force on practice guidelines. Circulation. 2014;129:2440-92.

13. Sasayama S, Asanoi H, Ishizaka S, Miyagi K. Evaluation of functional capacity of patients with congestive heart failure. In: Yasuda H, Kawaguchi H, editors. New aspects in the treatment of failing heart. Tokyo: SpringerVerlag; 1992. p. 113-7.

14. Norgren L, Hiatt WR, Dormandy JA, Nehler MR, Harris KA, Fowkes FG, et al. Inter-society consensus for the management of peripheral arterial disease (TASC II). Eur J Vasc Endovasc Surg. 2007. https://doi.org/10. 1016/j.ejvs.2006.09.024.

15. Kanda Y. Investigation of the freely available easy-to-use software "EZR" for medical statistics. Bone Marrow Transplant. 2013;48:452-8.

16. Chin CWL, Everett RJ, Kwiecinski J, Vesey AT, Yeung E, Esson G, et al. Myocardial fibrosis and cardiac decompensation in aortic stenosis. JACC Cardiovasc Imaging. 2017;10:1320-33.

17. Cohen LM, Ruthazer R, Moss AH, Germain MJ. Predicting six-month mortality for patients who are on maintenance hemodialysis. Clin J Am Soc Nephrol. 2010;5:72-9.

18. Lopes AA, Elder SJ, Ginsberg N, Andreucci VE, Cruz JM, Fukuhara S, et al. Lack of appetite in haemodialysis patients-associations with patient characteristics, indicators of nutritional status and outcomes in the international DOPPS. Nephrol Dial Transplant. 2007;22:3538-46.

19. Roshanravan B, Robinson-Cohen C, Patel KV, Ayers E, Littman AJ, de Boer $\mathrm{IH}$, et al. Association between physical performance and all-cause mortality in CKD. J Am Soc Nephrol. 2013;24:822-30.

\section{Publisher's Note}

Springer Nature remains neutral with regard to jurisdictional claims in published maps and institutional affiliations.
Ready to submit your research? Choose BMC and benefit from:

- fast, convenient online submission

- thorough peer review by experienced researchers in your field

- rapid publication on acceptance

- support for research data, including large and complex data types

- gold Open Access which fosters wider collaboration and increased citations

- maximum visibility for your research: over 100M website views per year

At BMC, research is always in progress.

Learn more biomedcentral.com/submissions 